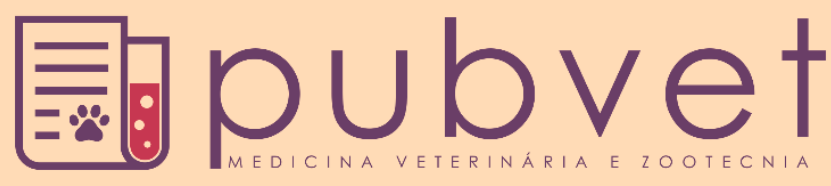

https://doi.org/10.31533/pubvet.v13n11a442.1-6

\title{
Desvio portossistêmico (shunt) intra-hepático em canino: relato de caso
}

\author{
Tairine Melo $\operatorname{Costa}^{1}{ }^{\ominus}$, Gilmara Muniz Baima ${ }^{2} \bullet$, Jéssica Maria da Silva Sousa ${ }^{2}$, Vanessa Brígida \\ Pinheiro Rodrigues $^{1}$, Thamara Barrozo Sampaio ${ }^{1}{ }^{\ominus}$, Eglésia Rodrigues Leite Fernandes ${ }^{1} \bullet$, Daniele \\ Benigno Mota $^{1}{ }^{\ominus}$, Leidiane Sousa Santos ${ }^{1} \bullet$, Iuliana Marjory Martins Ribeiro ${ }^{3}{ }^{\circ}$, Amanda da Costa

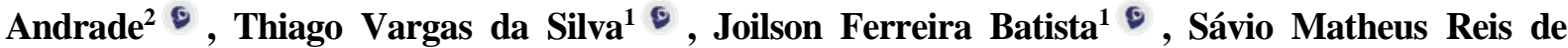 \\ Carvalho $^{10}$, Raylson Pereira de Oliveira ${ }^{4}{ }^{\circ}$, Márcia Paula Oliveira Farias ${ }^{5}{ }^{\circ}$, Francisco Lima Silva ${ }^{60}$ \\ ${ }^{1}$ Programa de Residência em Área Profissional da Saúde - Medicina Veterinária, Universidade Federal do Piauí-UFPI, Teresina, Piauí, Brasil; \\ ${ }^{2}$ Aprimoramento Profissional em Medicina Veterinária, Hospital Veterinário Universitário, Universidade Federal do Piauí- UFPI, Teresina, Piauí, Brasil; \\ ${ }^{3}$ Mestranda no Programa de Pós-Graduação em Tecnologias Aplicadas a Animais de Interesse Regional-PPGPAIR, Teresina, Piauí, Brasil; \\ ${ }^{4}$ Departamento de Medicina Veterinária, Universidade Federal Rural de Pernambuco-UFRPE, Pernambuco, Brasil \\ ${ }^{5}$ Professora do Campus Cinobelina Elvas/CPCE, Universidade Federal do Piauí-UFPI, Bom Jesus, Piauí, Brasil. \\ ${ }^{6}$ Professor Titular do Departamento de Clínica e Cirurgia veterinária, Universidade Federal do Piauí-UFPI, Teresina, Piauí, Brasil. \\ *Autor para correspondência: gilmaramuniz19@hotmail.com
}

Resumo. O presente relato tem como objetivo apresentar um caso de desvio portossistêmico (shunt) em um canino, macho, com dois meses de idade. Após dados sobre a anamnese, sinais clínicos, exame clínico e complementar, chegou-se ao diagnóstico de desvio portossistêmico intra-hepático. A ultrassonografia abdominal com Doppler foi fundamental para o diagnóstico, onde foi possível visualizar a presença de vaso anômalo entre artéria hepática e veia porta. Durante o protocolo terapêutico de estabilização do paciente para posterior correção cirúrgica, o animal veio a óbito.

Palavras chave: circulação sistêmica, desvio portossistêmico, ultrassonografia, veia porta

\section{Intrahepatic portosystemic shunt in canine: case report}

Abstract. This report aims to present a case of portosystemic shunt in a two-month-old male canine. After data on anamnesis, clinical signs, clinical and complementary examination, we arrived at the diagnosis of intrahepatic portosystemic shunting. Abdominal Doppler ultrasound was essential for the diagnosis, where it was possible to visualize the presence of anomalous vessel between the hepatic artery and the portal vein. During the patient stabilization protocol for subsequent surgical correction, the animal died.

Keywords: systemic circulation, portosystemic deviation, ultrasonography, portal vein

\section{Desviación portosistémica (shunt) intrahepático en canino: reporte de caso}

Resumen. Este relato tiene como objetivo presentar un caso de derivación portosistémica (shunt) en un canino macho de dos meses de edad. Después de los datos sobre anamnesis, signos clínicos, examen clínico y complementario, llegamos al diagnóstico de derivación portosistémica intrahepática. La ecografía Doppler abdominal fue esencial para el diagnóstico, donde fue posible visualizar la presencia de vasos anómalos entre la arteria hepática y la vena porta. Durante el protocolo de estabilización del paciente para la posterior corrección quirúrgica, el animal murió.

Palabras clave: circulación sistémica, desviación portosistémica, ultrasonografía, vena porta 


\section{Introdução}

O sistema hepático é responsável por diversas reações de bioconversão, irrigado pelo sistema arterial e venoso hepático e venoso portal, sendo a sua integridade anatômica indispensável para a funcionalidade do órgão (Baade et al., 2006). Na presença do desvio (shunt) de sangue venoso da circulação portal para a circulação sistêmica, ocorre o acúmulo de toxinas na circulação que seriam metabolizadas pelo fígado, causando um decréscimo nas concentrações de glicose, ureia, albumina, eritropoietina e colesterol (Fossum, 2014). Essas substâncias encontram-se circulantes e funcionam como falsos neurotransmissores, causando sinais neurológicos associados à encefalopatia hepática, agravados principalmente por ingestão de alimentos ricos em proteína (Fossum, 2014). São classificados como congênito ou adquirido e extra ou intra-hepático. Comumente os cães miniaturas são os mais acometidos, destacando-se as raças Schnauzer, Poodle, Shih-tzu, Yorkshire Terrier, Daschshund e Maltês. Algumas raças de gatos também podem ser acometidas (Reginatto et al., 2011).

Devido a insuficiência hepática o sistema nervoso manifesta sinais como ataxia, desorientação, andar em círculos, ausência de resposta a estímulos, cegueira, que pode ser episódica, principalmente em gatos (Mehl et al., 2005), convulsões e coma (Broome et al., 2004; Torisu et al., 2005; Winkler et al., 2003). O diagnóstico baseia-se no exame clínico, testes laboratoriais e exames por imagem, sendo necessário um conjunto de informações detalhadas para conclusão deste (Bonelli, 2011; Rothuizen \& Meyer, 2004).

O tratamento para a afecção inclui terapia médica, a correção cirúrgica dos vasos anastomosados, ou em algumas situações, a associação de ambas as terapias. A cirurgia consiste na ligação parcial ou completa do desvio e o tratamento clínico constitui no uso de antibióticos, lactulose e uma dieta com baixo teor de proteína (Faverzani et al., 2003).

Este trabalho tem como objetivo relatar um caso de desvio (shunt) portossistêmico em um canino, pois devido a sua importância na clínica médica de pequenos animais, busca-se contribuir para o seu correto diagnóstico e tratamento na clínica veterinária.

\section{Relato de caso}

Foi atendido no Hospital Veterinário da Universidade Federal do Piauí, Campus Agrícola da Socopo em Teresina - PI (HVU - UFPI), um canino, macho, raça Maltes, dois meses de idade, pesando 0,55 $\mathrm{kg}$. O animal chegou ao HVU passando por atendimento emergencial, onde a proprietária relata que adquiriu o animal de um canil localizado na cidade de Belo Horizonte-MG.

Durante a anamnese relatou ainda que o animal chegou alegre e agitado, oferecendo a este ração úmida (na qual posteriormente achou que estava estragada), pois pouco tempo depois de ingerir o alimento o mesmo ficou apático e apresentou episódios de diarreia durante o dia. No dia seguinte o animal foi levado a uma clínica veterinária na qual prescreveram antiprotozoário (antibioticoterapia sulfa + metronidazol) por via oral durante cinco dias, pois se suspeitou de giardíase. A tutora administrou o medicamento, mas relatou que não foi efetivo, retornando então a clínica, onde prescreveram Ampicilina por três dias e sem efetivo resultado o medicamento foi substituído por Doxiciclina.

O hemograma então foi solicitado e após o resultado foi sugerido internação do animal, que permaneceu na clínica por três dias. No período de internação o animal teve um episódio de convulsão. Sem sucesso no tratamento o animal foi encaminhado no dia seguinte para o HVU para o atendimento de urgência. Segundo a tutora, foi administrada a primeira dose da vacina viral; porém, não apresentou a carteira de vacinação. A mesma não trouxe os resultados dos exames anteriormente realizados.

No exame clínico observaram-se mucosas oculares, oral e peniana hipocoradas, orifício anal reduzido em diâmetro, secreção ocular, sem presença de dor abdominal, grau de desidratação 8\%, ausência de ectoparasitas, linfonodos não reativos, hipertermia, bradicardia, bradipneia, caquexia, anorexia e diarreia.

Primariamente foram solicitados os seguintes exames: Hemograma, bioquímicos, teste para detecção de antígeno para Cinomose (apresentando resultado negativo) e pesquisa de hemoparasitos (também negativa a amostra analisada). No Hemograma atualizado constataram-se alterações nos valores de Hemácia (2,5) (vr: 5,5-8,5), Hemoglobina (4,7 g/dL) (vr: 12-18 g/dL), Hematócrito (15\%) (vr: 37-55\%), CHGM $(31,3)$ (vr: 32-36), os quais se encontraram em valores abaixo do normal, já os valores de VGM 
(60) (vr: 60-77), RDW (\%) (19) (vr: 16-24) e plaquetas (483) (vr: 200-500), encontraram-se dentro dos valores considerados de referência. No Leucograma, os neutrófilos segmentados relativos (85) (vr:6077) e absolutos (11.645) (vr: 3.000-11.500) mostraram-se aumentados; linfócitos (8) (vr: 12-30) e eosinófilos diminuídos (0) (vr: 2-10). Outros parâmetros dentro dos valores de referência (Jain \& Jain, 1993; Meyer \& Harvey, 2004).

Nos resultados bioquímicos observou-se alterações nos valores de Ureia (19 mg/dL) (vr: 21,4-59,9 $\mathrm{mg} / \mathrm{dL}$ ), Creatinina (0,2 mg/dL) (vr: 0,5-1,5 mg/dL), ALT/TGP (15,0 U/L) (vr: 21,0-73,0 U/L), Proteína total $(3,2 \mathrm{~g} / \mathrm{dL})(\mathrm{vr}: 6,0-8,0 \mathrm{~g} / \mathrm{dL})$, Albumina $(1,5 \mathrm{~g} / \mathrm{dL})(\mathrm{vr}: 2,6-3,3 \mathrm{~g} / \mathrm{dL})$ e Globulina $(1,7 \mathrm{~g} / \mathrm{dL})$ (vr: 2,7-4,4 g/dL), encontrando-se com valores diminuídos e não havendo alterações nos outros parâmetros (Jain \& Jain, 1993; Kaneko et al., 2008; Meyer \& Harvey, 2004).

Após a análise dos exames optou-se por realizar a internação do animal e como terapia imediata, a transfusão sanguínea. Com o início da terapia suporte, o mesmo foi encaminhado ao setor de Diagnóstico por Imagem, para realização da ultrassonografia abdominal e pélvica com avaliação vascular por Doppler. O exame ultrassonográfico é realizado com transdutor setorial de 4,0-8,0 MHz. A varredura ultrassonográfica demonstrou (FAST). Na cavidade abdominal constatou-se presença de líquido livre em quantidade importante, com celularidade discreta, e com destaque para o recesso hepatorenal, esplênico e em região mesogástrica.

O fígado com silhueta hepática exibindo aumento, com a área hepática ultrapassando a curvatura maior do estômago. Vasos portais dilatados (congestão). Veia porta principal com dilatação evidente. Presença de fluxo turbulento. Velocidades de fluxo em veia porta direita reduzida, $<12 \mathrm{~cm} / \mathrm{s}$. O diagnóstico da imagem ultrassonográfica foi sugestivo de desvio portossistêmico intra-hepático, com presença de vaso anômalo entre artéria hepática e veia porta (Figura 1).
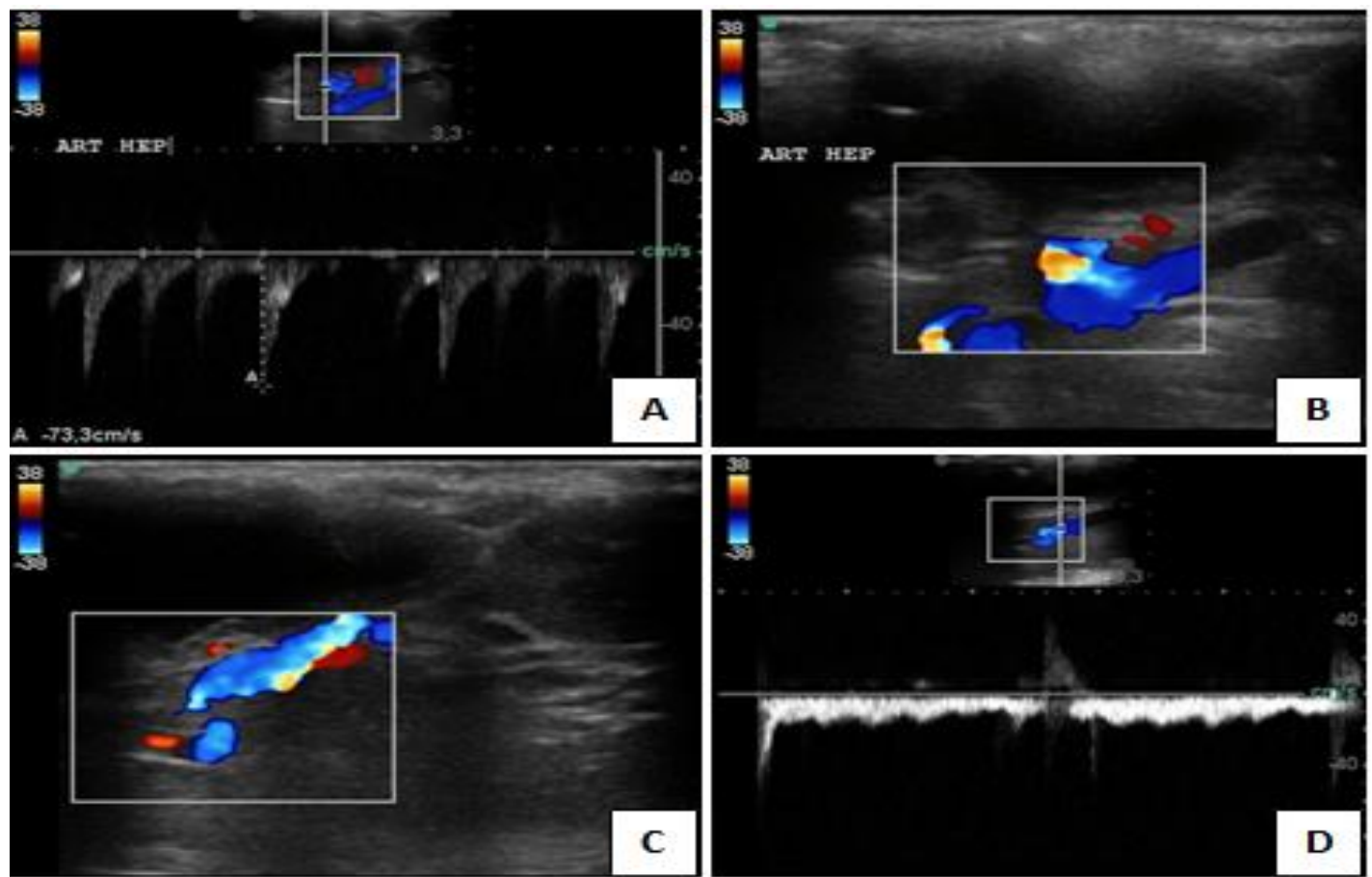

Figura 1. Imagens ultrassonográficas em modo espectral e color. (A) Artéria hepática exibindo fluxo arterial semiparabólico com leito vascular de resistividade intermediaria. Velocidade de pico sistólico (VPS): 73,3cm/s. (B) e (C) Imagens ultrassonográficas em modo color Doppler na comunicação entre artéria hepática e veia porta, representando artefato de aliasing (mosaico de cores). (D) Fluxo espectral venoso turbulento aferido em comunicação anômala entre artéria hepática e veia porta.

Durante o internamento para estabilização do paciente e posterior cirurgia para correção do desvio, administrou-se Cloridrato de Ranitidina injetável (2 mg/kg), Amoxicilina (15 mg/kg) e Solução Ringer com Lactato $250 \mathrm{ml}$. Após oito horas de internação, o animal veio a óbito, com suspeita do aumento do fluxo sanguíneo. Os achados de necropsia confirmaram os resultados dos exames ultrassonográficos e clínicos. 


\section{Resultados e discussão}

O desvio portossistêmico consiste em distúrbios circulatórios do fígado apresentando-se como vasos anômalos que permitem que o sangue portal normal que drena do estômago, baço, intestinos, e do pâncreas entre diretamente na circulação sistêmica, sem passar primeiramente pelo fígado (Fossum, 2014). Dessa forma, ocorre a acumulação de toxinas na circulação que seriam metabolizadas pelo fígado, causando um decréscimo nas concentrações de ureia, albumina, glicose, colesterol e eritropoietina (Fossum, 2014). A circulação destas substâncias funciona como falsos neurotransmissores, podendo causar sinais neurológicos associados à encefalopatia hepática, que são agravados principalmente por ingestão de alimentos ricos em proteína (Fossum, 2014).

A encefalopatia hepática é uma síndrome clínica do sistema nervoso central, que em decorrência da insuficiência hepática, altera a sua função. Uma variedade de substâncias (metionina/mercaptanos, ácidos graxos de cadeia curta, amônia, alterações na proporção entre os níveis circulantes de aminoácidos aromáticos e de cadeia ramificada, ácidos $\gamma$-aminobutírico) tem sido sugerida para resultar na elaboração de falsos neurotransmissores (Fossum, 2014).

A classificação de anomalias hepáticas vasculares é confusa e muitas vezes contraditória (Ettinger et al., 2017). Os desvios congênitos extra-hepáticos são tipicamente vasos anômalos individuais que permitem o fluxo sanguíneo anormal diretamente da veia porta para a circulação sistêmica, onde $63 \%$ dos casos são decorrentes em cães, mas também ocorrem em gatos (Fossum, 2014). Os desvios adquiridos ocorrem secundariamente a doenças hepáticas, como hepatites crônicas e cirrose hepática, resultando em hipertensão portal crônica (Tivers \& Lipscomb, 2011a, 2011b). Ainda no que se refere à classificação, podem ser solitários ou múltiplos (Havig \& Tobias, 2002). Corroborando com o animal relatado, os desvios intra-hepáticos geralmente são congênitos, havendo a comunicação da veia porta com a veia cava caudal por desvio da veia hepática esquerda. Isto ocorre devido ao não fechamento do ducto venoso fetal, representando cerca de 35\% desvios em cães e 10\% em gatos (Ettinger et al., 2017).

Segundo Reginatto et al. (2011), as raças mais predispostas são: Schnauzer, Poodle, Shih-tzu, Yorkshire Terrier, Daschshund e Maltês, ou seja, cães de pequeno porte, condizendo assim, com o animal descrito da raça Maltês.

O diagnóstico de doenças hepáticas, em geral, é baseado no exame clínico, testes laboratoriais e exames por imagem, e/ou laparotomia exploratória, sendo necessário um conjunto de informações detalhadas para conclusão do diagnóstico (Birchard \& Sherding, 2008; Bonelli, 2011; Rothuizen \& Meyer, 2004).

O paciente descrito apresentou alterações clínicas como: episódio de convulsão, mucosas hipocoradas, secreção ocular, grau de desidratação 8\%, hipertermia, bradicardia, bradipneia, caquexia, anorexia, diarreia, que condiz com Ettinger et al. (2017) onde os principais sinais clínicos relatados são bradicardia, perda de peso, febre e intolerância a anestésicos ou tranquilizantes. Alguns animais possuem crescimento menor do que o normal (Miller \& Fowler, 2006) ou possuem baixo escore corporal (Tivers \& Lipscomb, 2011a).

Episódios de distúrbios gastrointestinais também foram observados no animal relatado, conforme descrito por Ettinger et al. (2017) diante de casos mais graves pode-se observar alterações no sistema nervoso central, gastrointestinal e urinário. Quando há afecção cerebral difusa ocorre simultaneamente debilidade episódica, como ataxia, andar em círculos, letargia, alterações comportamentais, cegueira, convulsões e coma, condizendo com os sinais clínicos da encefalopatia hepática (Van Straten et al., 2015). Os sinais referentes com o trato urinário incluem poliúria, disúria, estrangúria, hematúria, obstrução uretral e formação de cristais de urato de amônia na urina. Os animais podem ter ainda polidipsia e poliúria (Broome et al., 2004; Fossum, 2014).

As alterações de creatinina, ALT/TPG, proteína total e albumina observadas no presente estudo estão de acordo com Nelson \& Couto (2015) que se refere a diminuição das concentrações de albumina, proteína total, ureia, colesterol e glicose e possível diminuição na síntese hepática. A diminuição na atividade da alanina transaminase, transaminase e fosfatase alcalina também podem ser detectadas, podendo ser considerada um sinal de insuficiência hepática (Center \& Magne, 1990). Segundo Tivers \& Lipscomb (2011a), a creatinina pode encontrar-se reduzida em reflexo à pouca massa muscular 
presente nestes animais. Relacionado ao hemograma observou-se anemia normocítica com eritrócitos, hipocrômica e arregenerativa grave, que de acordo com Meyer \& Harvey (2004), o hemograma pode evidenciar microcitose com eritrócitos normocrômicos, células em alvo e anemia arregenerativa leve.

O diagnóstico ultrassonográfico foi sugestivo para desvio portossistêmico intra-hepático, com presença de vaso anômalo entre artéria hepática e veia porta. Segundo Bright et al. (2006) a ultrassonografia é um exame confiável para detecção de desvios portossistêmico intra-hepático, não invasivo e com rara necessidade de utilização anestésica, onde o fígado normalmente aparece pequeno, apresenta hipovascularidade e o vaso do desvio pode ser identificado. Com isso, a ultrassonografia com Doppler colorido tem sido um método seguro de diagnóstico, auxiliando na localização do desvio, local da comunicação da turbulência (Carvalho \& Chammas, 2008; D'Anjou et al., 2004).

Segundo Tobias \& Johnston (2013), todos os animais com desvio portossistêmico são considerados pretendentes para cirurgia. Porém antes de submeter o animal ao procedimento cirúrgico observar as condições do paciente, iniciando um tratamento clínico para correção de líquidos, ganho de peso, taxa de glicemia, estado geral do animal e, principalmente, verificação de encefalopatia hepática, esse tratamento é feito por alguns dias até que o mesmo tenha condições de ser submetido a um protocolo anestésico e cirúrgico (Nelson \& Couto, 2015; Tobias \& Johnston, 2013).

Diante da necessidade de previamente estabilizar o paciente, optou-se primariamente pela terapia clínica, realizada conforme citado por Broome et al. (2004), com o uso de antibiótico Amoxicilina, segura para ser utilizada por um período prolongado sem ocasionar efeitos colaterais indesejáveis. Cita também o uso do metronidazol como uma boa escolha em exacerbação aguda de episódios de encefalopatias, no entanto apresenta um prognóstico ruim devido à deterioração contínua da função hepática. Em virtude de o fígado apresentar-se em muitos casos, insuficiente em casos de anomalia vascular e portossistêmica, deve-se ter muito cuidado com a anestesia, visto que muitos fármacos anestésicos são metabolizados por este (Butler et al., 1990). Apesar da tentativa de estabilização com a opção terapêutica, o paciente infelizmente veio a óbito antes do procedimento cirúrgico.

\section{Conclusão}

Desvio portossistêmico é uma afecção que apresenta sinais clínicos inespecíficos dificultando o seu diagnóstico. O diagnóstico tardio leva a gravidade da enfermidade dificultando o tratamento cirúrgico, no entanto, as considerações mais significativas sobre o manejo desta condição estão o uso de recursos clínicos ou cirúrgicos para reduzir ou prevenir a encefalopatia hepática.

\section{Referências bibliográficas}

Baade, S., Aupperle, H., Grevel, V. \& Schoon, H. A. (2006). Histopathological and immunohistochemical investigations of hepatic lesions associated with congenital portosystemic shunt in dogs. Journal of Comparative Pathology, 134(1):80-90.

Birchard, S. J. \& Sherding, R. G. (2008). Manual Saunders: clínica de pequenos animais (Vol. 3). São Paulo.

Bonelli, M. A. (2011). Shunt Portossistêmico em cães e gatos. Medicina Veterinária, 2(2):44-50.

Bright, S. R., Williams, J. M. \& Niles, J. D. (2006). Outcomes of intrahepatic portosystemic shunts occluded with ameroid constrictors in nine dogs and one cat. Veterinary Surgery, 35(3):300-309.

Broome, C. J., Walsh, V. P. \& Braddock, J. A. (2004). Congenital portosystemic shunts in dogs and cats. New Zealand Veterinary Journal, 52(4):154-162.

Butler, L. M., Fossum, T. W. \& Boothe, H. W. (1990). Surgical management of extrahepatic portosystemic shunts in the dog and cat. Paper presented at the Seminars in Veterinary Medicine and Surgery (Small Animal).

Carvalho, C. F. \& Chammas, M. C. (2008). Uso do ultrassom duplex Doppler no diagnóstico de shunt portossistêmico em gatos. Arquivo Brasileiro de Medecina Veterinária e Zootecnia, 60(1):109-112.

Center, S. A. \& Magne, M. L. (1990). Historical, physical examination, and clinicopathologic features of portosystemic vascular anomalies in the dog and cat. Paper presented at the Seminars in Veterinary Medicine and Surgery (Small Animal). 
D'Anjou, M. A., Penninck, D., Cornejo, L. \& Pibarot, P. (2004). Ultrasonographic diagnosis of portosystemic shunting in dogs and cats. Veterinary Radiology \& Ultrasound, 45(5):424-437.

Ettinger, S. J., Feldman, E. C. \& Cote, E. (2017). Textbook of Veterinary Internal Medicine-eBook. USA: Elsevier Health Sciences.

Faverzani, S., Trombetta, R., Grieco, V. \& Acocella, F. (2003). Clinical, laboratory, ultrasonographic and histopathological findings in dogs affected by portosystemic shunts, following surgery or medical treatment. Veterinary Research Communications, 27755-758.

Fossum, T. W. (2014). Cirurgia de pequenos animais (4 ed. Vol. 1). São Paulo: Elsevier Brasil.

Havig, M. \& Tobias, K. M. (2002). Outcome of ameroid constrictor occlusion of single congenital extrahepatic portosystemic shunts in cats: 12 cases (1993-2000). Journal of the American Veterinary Medical Association, 220(3):337-341.

Jain, N. C. \& Jain, A. H. (1993). Essentials of Veterinary Hematology (1st ed.). Davis, CA, USA: WileyBlackwell.

Kaneko, J. J., Harvey, J. W. \& Bruss, M. L. (2008). Clinical biochemistry of domestic animals (Vol. 1). New York: Academic press.

Mehl, M. L., Kyles, A. E., Hardie, E. M., Kass, P. H., Adin, C. A., Flynn, A. K., . . Gregory, C. R. (2005). Evaluation of ameroid ring constrictors for treatment for single extrahepatic portosystemic shunts in dogs: 168 cases (1995-2001). Journal of the American Veterinary Medical Association, 226(12):2020-2030.

Meyer, D. J. \& Harvey, J. W. (2004). Veterinary laboratory medicine: interpretation \& diagnosis. Philadelphia, USA: Sauders.

Miller, J. M. \& Fowler, J. D. (2006). Laparoscopic portosystemic shunt attenuation in two dogs. Journal of the American Animal Hospital Association, 42(2):160-164.

Nelson, R. W. \& Couto, C. G. (2015). Medicina interna de pequenos animais. Amsterdan: Elsevier Editora.

Reginatto, R. C., Frehse, M. S., Tanaka, N. M., Fávero, V., Sprea, G., Bach, F. S. \& Sanson, M. N. (2011). Shunt portossistêmico extra-hepático em cadela maltês de 8 meses. Semina: Ciências Agrárias, 32(2):739-746.

Rothuizen, J. \& Meyer, H. P. (2004). Anamnese, exame físico e sinais da doença hepática. In S. J. Ettinger \& A. Feldman (Eds.), Tratado de Medicina Interna Veterinária (Vol. 2, pp. 1342-1347). São Paulo, São Paulo, Brasil: Manole.

Tivers, M. \& Lipscomb, V. (2011a). Congenital portosystemic shunts in cats: investigation, diagnosis and stabilisation. Journal of Feline Medicine and Surgery, 13(3):173-184.

Tivers, M. \& Lipscomb, V. (2011b). Congenital portosystemic shunts in cats: surgical management and prognosis. Journal of Feline Medicine and Surgery, 13(3):185-194.

Tobias, K. M. \& Johnston, S. A. (2013). Veterinary Surgery: Small Animal-E-BOOK: 2-Volume Set. St. Louis, USA: Elsevier Health Sciences.

Torisu, S., Washizu, M., Hasegawa, D. \& Orima, H. (2005). Brain magnetic resonance imaging characteristics in $\operatorname{dogs}$ and cats with congenital portosystemic shunts. Veterinary Radiology \& Ultrasound, 46(6):447-451.

Van Straten, G., Spee, B., Rothuizen, J., Van Straten, M. \& Favier, R. P. (2015). Diagnostic value of the rectal ammonia tolerance test, fasting plasma ammonia and fasting plasma bile acids for canine portosystemic shunting. The Veterinary Journal, 204(3):282-286.

Winkler, J. T., Bohling, M. W., Tillson, D. M., Wright, J. C. \& Ballagas, A. J. (2003). Portosystemic shunts: diagnosis, prognosis, and treatment of 64 cases (1993-2001). Journal of the American Animal Hospital Association, 39(2):169-185.

Recebido: 3 de setembro, 2019.

Aprovado: 30 de outubro, 2019.

Publicado: 11 de novembro, 2019.

Licenciamento: Este artigo é publicado na modalidade acesso aberto sob a licença Creative Commons Atribuição 4.0 (CC-BY 4.0), a qual permite uso irrestrito, distribuição, reprodução em qualquer meio, desde que o autor e a fonte sejam devidamente creditados. 